\title{
Excitation functions of proton and deuteron induced reactions on iron and alpha-particle induced reactions on manganese in the energy region up to $25 \mathrm{MeV}$
}

\author{
S. Sudár ${ }^{1,2}$ and S. M. Qaim ${ }^{1}$ \\ ${ }^{1}$ Institut für Nuklearchemie, Forschungszentrum Jülich GmbH, D-52425 Jülich, Germany \\ ${ }^{2}$ Institute of Experimental Physics, Kossuth University, H-4001 Debrecen, Hungary
}

(Received 1 July 1994)

\begin{abstract}
Excitation functions were measured by the stacked-foil technique for ${ }^{\text {nat }} \mathrm{Fe}(p, x n)^{56} \mathrm{Co}$, ${ }^{\text {nat }} \mathrm{Fe}(p, x n){ }^{57} \mathrm{Co}, \quad{ }^{58} \mathrm{Fe}(p, n){ }^{58} \mathrm{Co}^{m+g}, \quad{ }^{57} \mathrm{Fe}(p, \alpha){ }^{54} \mathrm{Mn}, \quad{ }^{\text {nat }} \mathrm{Fe}(d, x n){ }^{58} \mathrm{Co}^{m+g}, \quad{ }^{56} \mathrm{Fe}(d, 2 n){ }^{56} \mathrm{Co}$, ${ }^{56} \mathrm{Fe}(d, \alpha){ }^{54} \mathrm{Mn},{ }^{55} \mathrm{Mn}(\alpha, n){ }^{58} \mathrm{Co}^{m+g}$, and ${ }^{55} \mathrm{Mn}(\alpha, 2 n){ }^{57} \mathrm{Co}$ reactions from the respective thresholds to $14.12 \mathrm{MeV}$ in work with protons, $12.97 \mathrm{MeV}$ with deuterons, and $25.52 \mathrm{MeV}$ with alpha particles. The radioactivity of the activation products was determined by high resolution $\gamma$-ray spectroscopy. Statistical model calculations taking into account the precompound effects were performed for all the reactions, using a consistent set of model parameters. The experimental excitation functions of the proton and alpha-particle induced reactions are described within 10 to 15 percent by the calculation over the whole investigated energy range. The shapes of the excitation functions of the deuteron induced reactions are also reproduced well by the model calculation. However, a reduction factor $(R=0.56)$ is needed to take account of the direct breakup process and, thereby, to obtain good agreement between the experimental data and model calculations.
\end{abstract}

PACS number(s): 24.60.Dr, 25.40.-h, 25.45.-z, 25.55.-e

\section{INTRODUCTION}

Studies of excitation functions of charged particle induced reactions are of considerable significance for testing nuclear models and for practical applications, especially in cyclotron production of radioisotopes and astrophysics. Of particular interest are investigations on nuclear reactions induced by various projectiles (protons, deuterons, $\alpha$ particles, etc.) but leading to the same product nuclei. This way the role of nuclear structure effects is diminished, and the effect of input nuclear model parameters can be investigated.

We chose to study the formation of ${ }^{56} \mathrm{Co},{ }^{57} \mathrm{Co}$, ${ }^{58} \mathrm{Co}^{m+g}$, and ${ }^{54} \mathrm{Mn}$ in several processes. The radioisotopes ${ }^{57} \mathrm{Co}$ and ${ }^{54} \mathrm{Mn}$ are commonly used in $\gamma$-ray spectroscopy as standards; the former also in Mössbauer spectroscopy. A knowledge of the various excitation functions, especially near the reaction thresholds, should help in the optimization of the production processes at small cyclotrons. The major goal, however, was to test the applicability of nuclear model calculations over an energy range up to about $25 \mathrm{MeV}$.

The most commonly used method for excitation function measurements consists of the stacked-foil technique. There are two major sources of errors in this technique: (a) beam current measurement via monitor reactions and (b) mean particle energy determination in a thick sample. The latter needs special attention near the reaction threshold. We considered the two points in detail in this work.

\section{EXPERIMENTAL}

Excitation functions were measured using the stackedfoil technique, described in several publications from Jülich [cf. 1-3]. Some of the important features relevant to the present work are given below.

\section{A. Composition of the stack and purity of foils}

High purity thin iron foils $(99.95 \%, 25 \mu \mathrm{m}$, supplied by Goodfellow Metals, England) were used as samples for the irradiations with protons and deuterons. For investigations of $\alpha$-particle induced reactions on manganese thin foils $(20 \mu \mathrm{m})$ of a special $\mathrm{Mn} / \mathrm{Ni}$ alloy were used, pure manganese foils being not available. The exact composition of the alloy was $\mathrm{Mn}: 87.10 \pm 0.05 \%$, $\mathrm{Ni}: 12.89 \pm 0.05 \%$, and Fe: $0.018 \pm 0.002 \%$. It was determined by optical emission spectroscopy-inductively coupled plasma (OES-ICP) analysis.

To check the incident particle energy, the energy degradation in the stack, and the beam intensity, appropriate monitor foils were inserted in each stack. The foils used were as follows: $\mathrm{Cu}$ and $\mathrm{Ni}$ for the proton beam, $\mathrm{Al}$ for deuteron beam, and $\mathrm{Cu}$ for the alpha-particle beam. All the foils used were cut from high purity materials $(99.95 \%)$ of different thickness, thereby allowing changes in the mean energies of the irradiated $\mathrm{Fe}$ or $\mathrm{Mn} / \mathrm{Ni}$ samples.

\section{B. Irradiations and mean particle energies}

Each stack was irradiated for 20 to 30 minutes using the external beam at the Compact Cyclotron CV28 of the KFA Jülich. The primary proton energies used were 14.87 MeV and $18.61 \mathrm{MeV}$; in the case of deuterons it was $13.40 \mathrm{MeV}$ and for alpha particles $26.70 \mathrm{MeV}$. The energy of each particle beam was measured by a time of flight method [4] before or after the irradiation and had an uncertainty of $\pm 0.15 \mathrm{MeV}$. Four stacks were irradiated for work on each target element with a particular incident particle.

Starting with the primary particle energy incident on the front foil of the stack, the particle energy $(\bar{E})$ effec- 
TABLE I. Monitor reactions used for checking the beam current and energy degradation.

\begin{tabular}{|c|c|}
\hline Particle beam & Reactions [Ref.] \\
\hline Proton & $\begin{array}{c}{ }^{\text {nat }} \mathrm{Ni}(p, x){ }^{57} \mathrm{Co}[38] ;{ }^{\text {nat }} \mathrm{Ni}(p, x){ }^{55} \mathrm{Co}[38] \\
{ }^{\text {nat }} \mathrm{Ni}(p, x){ }^{57} \mathrm{Ni}[38] ;{ }^{63} \mathrm{Cu}(p, n){ }^{63} \mathrm{Zn}[39] ; \\
{ }^{65} \mathrm{Cu}(p, n){ }^{65} \mathrm{Zn}[40]\end{array}$ \\
\hline $\begin{array}{l}\text { Deuteron } \\
\text { Alpha }\end{array}$ & $\begin{array}{c}{ }^{56} \mathrm{Fe}(d, n){ }^{57} \mathrm{Co}[32] ;{ }^{27} \mathrm{Al}(d, x){ }^{24} \mathrm{Na}[41] \\
{ }^{63} \mathrm{Cu}(\alpha, n){ }^{66} \mathrm{Ga}[42] ;{ }^{63} \mathrm{Cu}(\alpha, n p){ }^{65} \mathrm{Zn}[43]\end{array}$ \\
\hline
\end{tabular}

tive at each sample was obtained using the range-energy formula [cf. 5], taking a mean of the energies of the ingoing and outgoing particles. This approximation for the effective particle energy is valid only if the foil is thin or the excitation function of the investigated reaction is approximately constant over the energy range covered by the foil. In general, the measured mean cross section $(\bar{\sigma})$ can be expressed in the following form:

$$
\bar{\sigma}=\frac{1}{d} \int_{E_{1}}^{E_{2}} \sigma(E) \frac{1}{(d E / d x)} d E,
$$

where $d, E_{1}, E_{2}, \sigma(E)$, and $(d E / d x)$ are the thickness of the foil, the incident and outgoing energy of the beam, the cross section of the investigated reaction as a function of the energy and the specific energy loss of the projectile in the foil, respectively. Generally $\bar{\sigma}$ is not equal to $\sigma(\bar{E})$, but there exists an energy $\hat{E}$ for which $\bar{\sigma}=\sigma(\hat{E})$, where $E_{1}<\hat{E}<E_{2}$. The $\hat{E}$ was estimated by using the excitation functions of the investigated reactions derived from the model calculations in the following way: A value $\bar{\sigma}_{\text {th }}$ was derived from the calculated cross sections, and the $\hat{E}$ was interpolated to fulfill the $\bar{\sigma}_{\text {th }}=\sigma_{\text {th }}(\hat{E})$ equation. This method gave significant differences, compared with the usual simple estimation method for obtaining the mean energy, only near the reaction threshold. Its contribution was very important in the case of alphaparticle induced reactions where the energy loss of the beam was much higher than in the case of protons and deuterons.

The energy degradation along the stack and the beam current were checked by the reactions induced in the inserted monitor foils. The reactions used are shown in Table I. The beam intensity was calculated in every monitor position using all the available monitor reactions and these "currents" were reflected in the primary particle energy incident in front of the stack. The beam current was calculated as the mean of the individually calculated currents, and the primary particle energy was determined to get the minimal $\chi^{2}$ for the beam current taking into account the errors of the measured activity and the used cross sections. Besides monitor foils the charge collected in a Faraday cup was also registered and from there the average beam current was deduced. The results generally agreed within $\pm 10 \%$.

\section{Measurement of radioactivity}

The activity of each sample was determined by $\gamma$ ray spectroscopy using a $\mathrm{Ge}(\mathrm{Li})$ detector coupled to an
ORTEC (Spectrum ACE) 4K MCA Plug-In Card at Jülich and a similar system with HPGe detector at Debrecen. The cards were connected to IBM-compatible PC-AT computer. The peak area analysis was done using the PC version of the GAMANAL [6,7] spectrum analyzer program.

Each sample was counted several times to check the half-life of each product. The initial activity was determined by least-squares fitting to the measured counts as a function of time using the half-life given in the literature.

The counting efficiencies of the detectors for various counting distances and photon energies were determined using calibrated standard sources, obtained from PTB Braunschweig, Amersham International, and Hungarian National Bureau of Standards.

\section{Calculation of cross sections and their errors}

The count rates were corrected for random pileup losses (using a pulse generator as reference) as well as for $\gamma$-ray abundance and the efficiency of the detector. Correction for coincidence loss was also taken into account. The most important decay data are summarized in Table II. Cross sections were calculated using the usual activation formula.

The major sources of errors involved were those associated with the measurement of the beam current and determination of the absolute activities of the products. The error in the excitation function of an individual monitor reaction was taken as $\mathbf{6 - 1 0 \%}$. The beam current was determined by a fitting procedure (see above) using several monitor reactions. Therefore the error in the beam current was adopted as $4-7 \%$, assuming that no correlation existed in the data of the different authors. Within the limits of error the calculated current was in good agreement with the current measured by the integrator. To do a complete error analysis it is necessary to know a covariance matrix of the reference data. This is unfortunately not available. The efficiency of the $\gamma$-ray detector was known within an uncertainty of 3-5\%. Some samples were measured both at Jülich and Debrecen and the agreement between the mesured activities was within $2-3 \%$, proving that the efficiency calibration procedures were reliable. The errors in the calculation of the abso-

TABLE II. Decay data of measured reaction products, taken from Browne and Firestone [44].

\begin{tabular}{cccc}
\hline \hline $\begin{array}{c}\text { Radio- } \\
\text { isotope }\end{array}$ & Half-life & $\begin{array}{c}\text { Gamma-ray } \\
\text { energy (keV) }\end{array}$ & $\begin{array}{c}\text { Gamma-ray } \\
\text { abundance (\%) }\end{array}$ \\
\hline${ }^{58} \mathrm{Co}$ & $70.92 \mathrm{~d}$ & 810.8 & 99.43 \\
${ }^{57} \mathrm{Co}$ & $271.77 \mathrm{~d}$ & 122.06 & 85.5 \\
& & 136.47 & 10.69 \\
${ }^{56} \mathrm{Co}$ & $77.7 \mathrm{~d}$ & 846.81 & 99.9 \\
& & 1238.31 & 67.0 \\
${ }^{54} \mathrm{Mn}$ & $312.2 \mathrm{~d}$ & 834.83 & 99.97 \\
\hline \hline
\end{tabular}

Each radioisotope decays $100 \%$ by EC. 
lute activity were: the error of the initial count rate at the end of the irradiation was determined by the leastsquares fitting procedure (see above), and it was about $0.5-25 \%$; the error in the decay data used was $<1 \%$ and that in the coincidence losses $<0.5 \%$. The error in the target atoms $/ \mathrm{cm}^{2}$ was $0.50-1.5 \%$. The total error in each cross section was obtained by combining the individual errors in quadrature.

\section{NUCLEAR MODEL CALCULATIONS}

Cross section calculations were done using the statistical model taking into account the preequilibrium effects. The calculation code STAPRE [8] was used. Direct interactions were not considered but their contribution should be $<10 \%$ in the case of proton and alpha-particle induced reactions. Neutron, proton, deuteron, and alpha-particle emissions were taken into account, and the transmission coefficients for these particles were calculated by the optical model code SCAT-2 [9]. The parameters for the optical model (OM) were chosen from a global parameter set. For the neutron the OM parameter set of Becchetti and Greenlees [10], while for proton and deuteron those of Perey [11] were used. In the case of alpha particles a modified set of the OM parameters of McFadden and Satchler [12] (modified by Uhl et al. [13]) was used. For the energy and mass dependence of the effective matrix element, $|M|^{2}=F_{M} A^{-3} E^{-1}$ formula was used with the value of $F_{M}=500$. The separation energies of the emitted particles were taken from Ref. [14]. The initial exciton number $n_{0}\left(p_{0}, h_{0}\right)$ has a strong influence on the calculated excitation function. The used initial particle and hole numbers were the usual values $p_{0}=2, h_{0}=1$ for protons while in the case of alpha as incident particles the $p_{0}=5, h_{0}=1$ were used [15]. In the case of deuteron the initial particle and hole numbers are more ambiguous in the literature. Therefore we tried to find those values which reproduce best the shapes of the measured excitation functions. These numbers were found to be $p_{0}=1, h_{0}=1$. The alpha emission was treated in the framework of Milazzo-Colli and Braga-Marcazzan model [16] with an alpha preformation factor of 0.25 .

The energies, spins, parities, and branching ratios of the discrete levels were selected from the Nuclear Data Sheets [17]. In the continuum region the level density was calculated by the back-shifted formula [18] with the level density parameter given in Ref. [18]. In cases where these parameters were not available they were estimated from the systematics and from the values of the neighboring isotopes. Occasionally, the level density parameters a and $\Delta$ were varied within their uncertainties to check their effect on the cross sections. The spin distribution of the level density was characterized by the ratio of the effective moment of inertia $\Theta_{\text {eff }}$ to rigid body moment of inertia $\Theta_{\text {rig }}\left(\eta=\Theta_{\text {eff }} / \Theta_{\text {rig }}\right)$ and the calculations were performed for $\eta=1.0$. The transmission coefficients of photons were calculated from the gamma-ray strength functions. For the $E 1$ radiation the Brink-Axel model with global parameters, while for the $M 1, E 2, M 2, E 3$, and $M 3$ radiation the Weisskopf model was used.

\section{RESULTS AND DISCUSSION}

\section{A. Proton induced reactions on iron}

The measured cross section data for the proton induced reactions ${ }^{\text {nat }} \mathrm{Fe}(p, x n){ }^{56} \mathrm{Co}$, nat $\mathrm{Fe}(p, x n){ }^{57} \mathrm{Co}$, ${ }^{58} \mathrm{Fe}(p, n){ }^{58} \mathrm{Co}^{m+g}$, and ${ }^{57} \mathrm{Fe}(p, \alpha){ }^{54} \mathrm{Mn}$ are summarized in Table III. The data are given for natural composition except for the ${ }^{58} \mathrm{Fe}(p, n){ }^{58} \mathrm{Co}^{m+g}$ reaction, since ${ }^{58} \mathrm{Co}$ was produced only by this reaction. Taking into account the isotopic composition of iron and the estimated cross sections for the competing reactions, the excitation functions of the ${ }^{56} \mathrm{Fe}(p, n){ }^{56} \mathrm{Co},{ }^{57} \mathrm{Fe}(p, n){ }^{57} \mathrm{Co}$, and ${ }^{57} \mathrm{Fe}(p, \alpha){ }^{54} \mathrm{Mn}$ reactions were also deduced over the investigated energy range.

The excitation function of the ${ }^{56} \mathrm{Fe}(p, n){ }^{56}$ Co reaction is shown in Fig. 1 together with data of Jenkins and

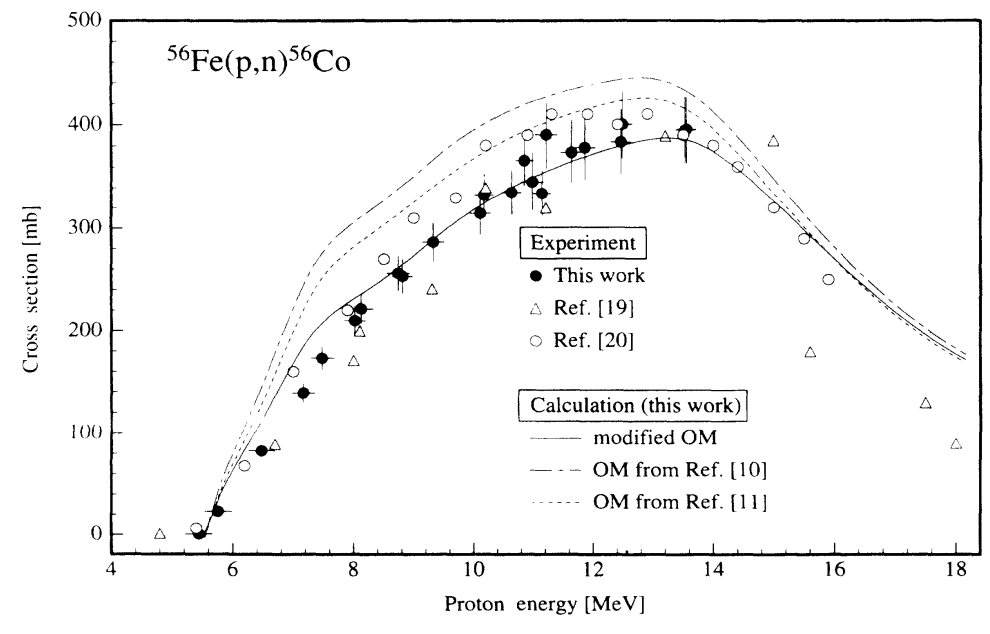

FIG. 1. Measured and calculated cross sections of the ${ }^{56} \mathrm{Fe}(p, n){ }^{56} \mathrm{Co}$ reaction. The experimental data were deduced from measurements on ${ }^{\text {nat }} \mathrm{Fe}$, i.e., the contribution of the ${ }^{57} \mathrm{Fe}(p, 2 n){ }^{56} \mathrm{Co}$ process was subtracted. The nuclear model calculation was done using three sets of OM parameters. 


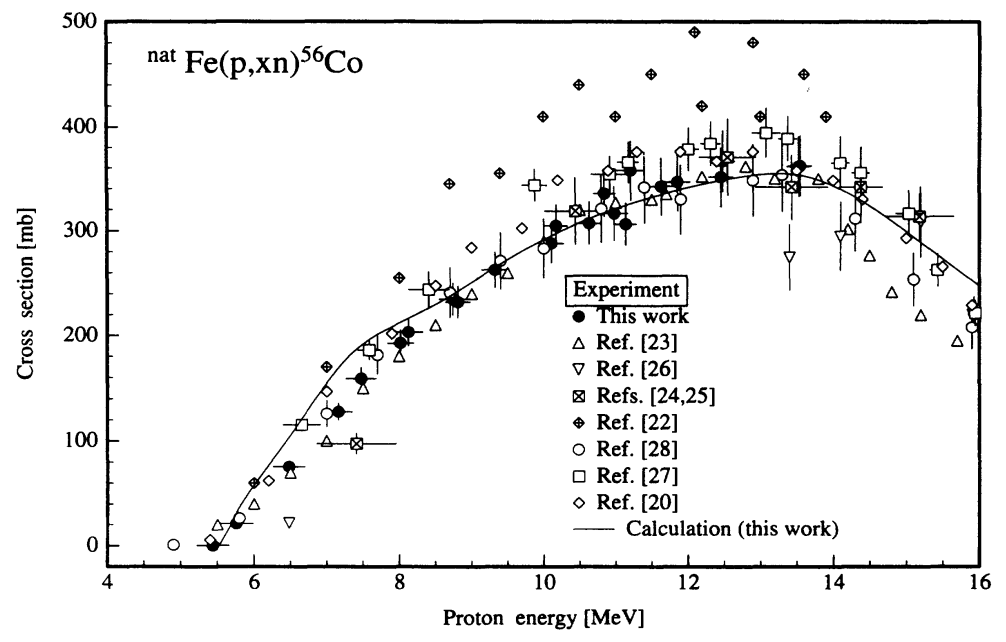

FIG. 2. Measured and calculated cross sections of the ${ }^{\text {nat }} \mathrm{Fe}(p, x n){ }^{56} \mathrm{Co}$ process. The nuclear model calculation was done using the modified OM parameters (see text).

Wain [19] and Dyer et al. [20]. Unfortunately error data were not reported in several older experiments. Therefore those data are plotted without erorr bars in all the figures. From the model calculation the contribution of the ${ }^{57} \mathrm{Fe}(p, 2 n){ }^{56} \mathrm{Co}$ process was found to be $<2 \%$ up to $18 \mathrm{MeV}$ proton energy; the measured data could thus be converted to single isotope cross sections. Figure 1 shows that the lowest energy point of Jenkins and Wain [19] is below the threshold of the reaction and is possibly due to uncertainties in the mean energy determination. In general, however, the deviations among the three data sets are not more than $20 \%$.

As far as the nat $\mathrm{Fe}(p, x n){ }^{56} \mathrm{Co}$ data are concerned, several measurements have been reported in the literature [21-28]. Our data together with all the other results are shown in Fig. 2. Our values are in agreement within the experimental errors with most of the data sets [20,23-25,27,28]. A significant deviation was found only with the data of Tanaka and Furukawa [22]. Those early measurements are 10-30\% higher than our values. Shoen

TABLE III. Measured cross sections of proton induced reactions on iron.

\begin{tabular}{|c|c|c|c|c|c|c|c|c|c|}
\hline \multirow{2}{*}{$\begin{array}{l}\text { Proton } \\
\text { energy } \\
(\mathrm{MeV})\end{array}$} & \multirow{2}{*}{$\begin{array}{c}\text { Error in } \\
\text { proton } \\
\text { energy } \\
(\mathrm{MeV})\end{array}$} & \multicolumn{2}{|c|}{${ }^{58} \mathrm{Fe}(p, n)^{58} \mathrm{Co}^{m+g}$} & \multicolumn{2}{|c|}{${ }^{\text {nat }} \mathrm{Fe}(p, x n){ }^{57} \mathrm{Co}$} & \multicolumn{2}{|c|}{${ }^{\mathrm{nat}} \mathrm{Fe}(p, x n){ }^{56} \mathrm{Co}$} & \multicolumn{2}{|c|}{${ }^{57} \mathrm{Fe}(p, \alpha){ }^{54} \mathrm{Mn}$} \\
\hline & & $\begin{array}{c}\sigma \\
(\mathrm{mb})\end{array}$ & $\begin{array}{c}\Delta \sigma \\
(\mathrm{mb})\end{array}$ & $\begin{array}{c}\sigma \\
(\mathrm{mb})\end{array}$ & $\begin{array}{c}\Delta \sigma \\
(\mathrm{mb})\end{array}$ & $\begin{array}{c}\sigma \\
(\mathrm{mb})\end{array}$ & $\begin{array}{c}\Delta \sigma \\
(\mathrm{mb})\end{array}$ & $\begin{array}{c}\sigma \\
(\mathrm{mb})\end{array}$ & $\begin{array}{c}\Delta \sigma \\
(\mathrm{mb})\end{array}$ \\
\hline 3.54 & 0.21 & 27.8 & 3.0 & 1.13 & 0.09 & & & & \\
\hline 4.90 & 0.2 & 164 & 11 & 3.83 & 0.26 & & & & \\
\hline 5.44 & 0.22 & & & & & 0.21 & 0.02 & & \\
\hline 5.71 & 0.23 & 346 & 51 & 6.22 & 0.75 & & & & \\
\hline 5.76 & 0.23 & & & & & 20.9 & 1.4 & & \\
\hline 6.48 & 0.22 & 455 & 35 & 7.40 & 0.53 & 75 & 5 & & \\
\hline 7.16 & 0.19 & 522 & 43 & 8.90 & 0.68 & 127 & 8 & & \\
\hline 7.48 & 0.21 & 524 & 45 & 8.97 & 0.65 & 158 & 10 & & \\
\hline 8.13 & 0.2 & 619 & 47 & 9.35 & 0.70 & 202 & 13 & & \\
\hline 8.02 & 0.18 & 546 & 54 & 9.67 & 0.79 & 192 & 13 & & \\
\hline 8.74 & 0.19 & 656 & 48 & 9.85 & 0.70 & 234 & 15 & & \\
\hline 8.81 & 0.17 & 584 & 41 & 10.24 & 0.71 & 231 & 15 & & \\
\hline 9.32 & 0.18 & 546 & 80 & 9.18 & 1.1 & 262 & 17 & & \\
\hline 10.17 & 0.16 & 667 & 55 & 11.15 & 0.80 & 304 & 20 & & \\
\hline 10.11 & 0.17 & 753 & 88 & 10.22 & 1.1 & 287 & 19 & & \\
\hline 10.63 & 0.16 & 819 & 121 & 9.14 & 1.1 & 306 & 20 & & \\
\hline 10.84 & 0.15 & 723 & 56 & 10.80 & 0.79 & 334 & 22 & & \\
\hline 10.98 & 0.19 & 643 & 69 & 11.17 & 0.97 & 315 & 26 & 25.1 & 5.4 \\
\hline 11.14 & 0.15 & 740 & 97 & 9.95 & 1.2 & 305 & 20 & & \\
\hline 11.21 & 0.19 & 741 & 58 & 10.81 & 0.81 & 356 & 29 & 24.8 & 5.1 \\
\hline 11.63 & 0.18 & 710 & 56 & 10.12 & 0.76 & 341 & 28 & 26.2 & 5.4 \\
\hline 11.86 & 0.18 & 754 & 59 & 9.59 & 0.72 & 345 & 28 & 29.3 & 5.4 \\
\hline 12.46 & 0.17 & 684 & 54 & 8.63 & 0.65 & 350 & 28 & 31.3 & 4.8 \\
\hline 12.48 & 0.17 & 705 & 55 & 8.45 & 0.64 & 366 & 30 & 28.7 & 6.0 \\
\hline 13.53 & 0.16 & 566 & 45 & 7.17 & 0.54 & 360 & 29 & 34.3 & 4.3 \\
\hline 13.55 & 0.16 & 543 & 43 & 6.67 & 0.51 & 361 & 29 & 37.2 & 6.6 \\
\hline 14.09 & 0.15 & 488 & 38 & 6.67 & 0.55 & 335 & 27 & 39.6 & 10.7 \\
\hline 14.12 & 0.15 & 458 & 36 & 6.40 & 0.48 & 344 & 28 & 35.0 & 4.1 \\
\hline
\end{tabular}




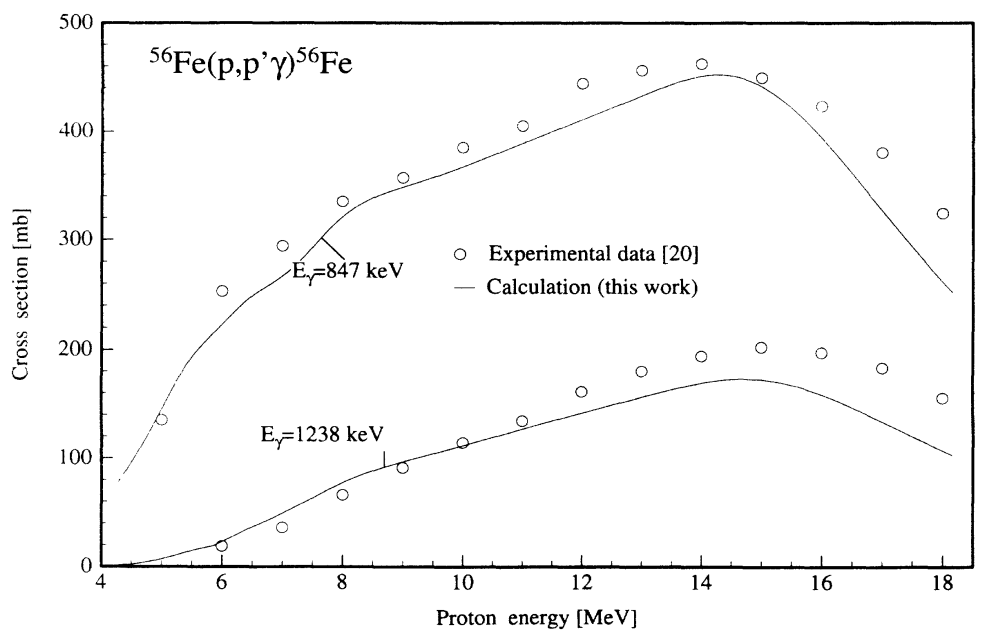

FIG. 3. Measured and calculated cross sections of the ${ }^{56} \mathrm{Fe}\left(p, p^{\prime} \gamma\right){ }^{56} \mathrm{Fe}$ reaction. The results refer to the population of two excited states in the product nucleus which emit two different prompt $\gamma$ rays (847 and $1238 \mathrm{keV}$ ).

et al. [26] reported only a few points in this energy range; those data appear to be significantly lower than the other data.

First calculations using the code STAPRE and the global OM parameters overestimated the cross sections over the whole energy range. Since below $8 \mathrm{MeV}$ proton energy only the contribution of the discrete levels is important, the systematic deviation either arose from the wrong choice of OM parameters or was caused due to occurrence of direct processes which make the compound absorption cross section unequal to the OM cross section. The calculated results of three OM parameter sets are given in Fig. 1. Using experimental results of Dyer et al. [20] on the ${ }^{56} \mathrm{Fe}\left(p, p^{\prime} \gamma\right)$ process it was possible to estimate the experimental absorption cross section and to modify the OM parameters to get better agreement with the experimental data. The thus modified parameters set gave a better agreement for the ${ }^{56} \mathrm{Fe}(p, n){ }^{56} \mathrm{Co}$ reaction (cf. Fig. 1), but the calculated cross sections are still somewhat higher in the 6-8 MeV region than the measured data. The calculated results given in Fig. 2 are also based on the modified OM parameters. The modified OM parameters for proton were the following: Real part of the local potential was of SaxonWoods form, $V_{0}=58.384-0.55 E \mathrm{MeV} ; r_{0}=1.25 \mathrm{fm}$; $a_{0}=0.65 \mathrm{fm}$; the surface peaked imaginary part was of
Saxon-Woods derivative form $W_{s}=13.5-0.15 E \mathrm{MeV}$; $r_{0}=1.25 \mathrm{fm} ; a_{0}=0.47 \mathrm{fm}$.

Dyer et al. [20] measured the excitation function of the ${ }^{56} \mathrm{Fe}\left(p, p^{\prime} \gamma\right){ }^{56} \mathrm{Fe}$ reaction using three prompt gamma rays. The results for the two strongest $\gamma$ lines are shown in Fig. 3. We performed calculations using the modified OM parameters and those results are also given in Fig. 3. Both the excitation functions are reproduced well by the model calculation. Apparently, the excitation functions can be described well by the model calculation if suitable OM model parameters are used. In this connection it would be important to do a detailed analysis to get a more reliable absorption cross section in the low energy region.

The cross sections for the ${ }^{\text {nat }} \mathrm{Fe}(p, x n)^{57} \mathrm{Co}$ process measured in this work and those reported in Refs. [24-27] are shown in Fig. 4. The results of model calculations using the modified OM parameters (see above) are also given and are in agreement with our experimental data, except near the threshold. The experimental data reported in Refs. [24-26] are in good agreement with our measurement above $10 \mathrm{MeV}$, but not below $10 \mathrm{MeV}$. The values given in Ref. [27], however, are systematically lower than the other measurements. The discrepancy is difficult to explain since the data for both ${ }^{56} \mathrm{Co}$ and ${ }^{57} \mathrm{Co}$ were obtained from the same foils: in the first case the

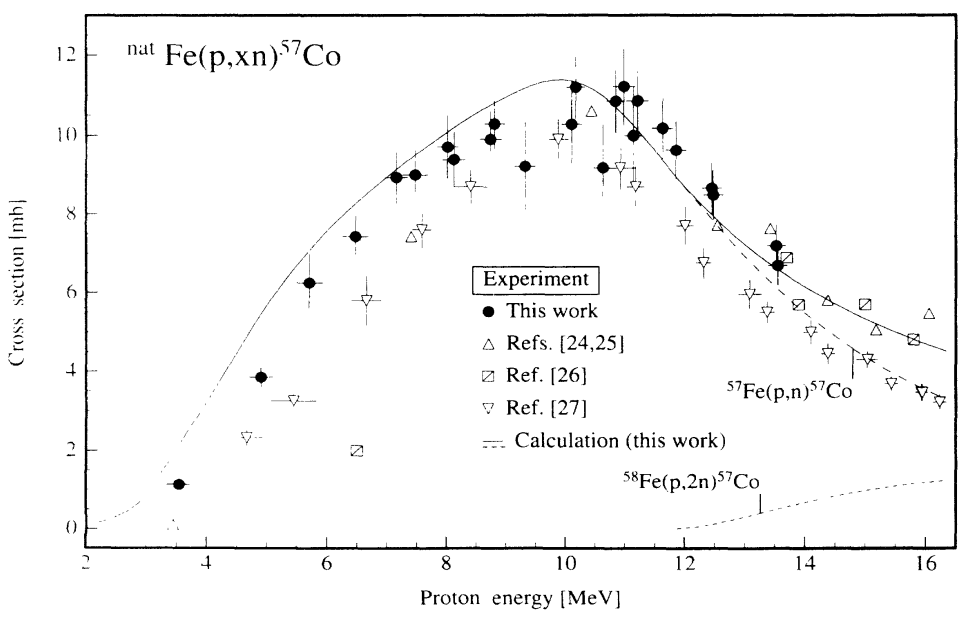

FIG. 4. Measured and calculated cross sections of the ${ }^{\text {nat }} \mathrm{Fe}(p, x n)^{57} \mathrm{Co}$ process. The contribution of the ${ }^{58} \mathrm{Fe}(p, 2 n)^{57}$ Co process obtained by calculation is also shown. 


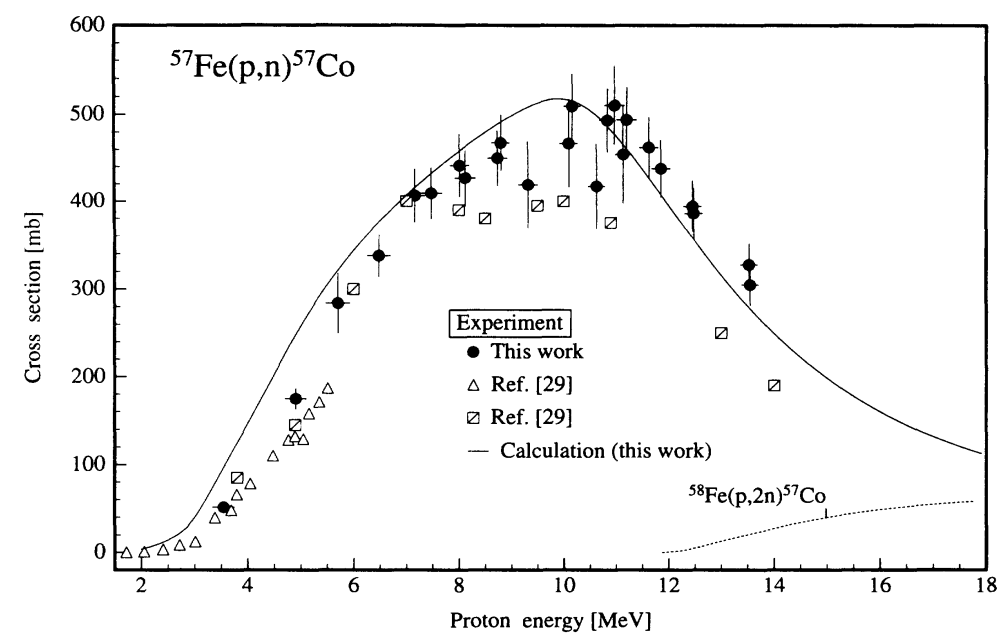

FIG. 5. Measured and calculated cross sections of the ${ }^{57} \mathrm{Fe}(p, n){ }^{57} \mathrm{Co}$ reaction. The experimental data were deduced from measurements on ${ }^{\text {nat }} \mathrm{Fe}$ by subtracting the contribution of the ${ }^{58} \mathrm{Fe}(p, 2 n)^{57} \mathrm{Co}$ process. cross sections are higher, but in the second lower. This could possibly arise from an error in the efficiency calibration of the $\mathrm{Ge}(\mathrm{Li})$ detector.

The dotted line in Fig. 4 represents the contribution of the ${ }^{58} \mathrm{Fe}(p, 2 n){ }^{57} \mathrm{Co}$ reaction to the total ${ }^{57} \mathrm{Co}$ formation, taking into account the isotopic composition of ${ }^{58} \mathrm{Fe}$ in natural iron. The contribution is negligible below $12 \mathrm{MeV}$ proton energy; our cross section data can thus be converted into the ${ }^{57} \mathrm{Fe}(p, n){ }^{57} \mathrm{Co}$ reaction cross sections using a small correction above $12 \mathrm{MeV}$. The results are shown in Fig. 5. The data reported in the literature $[22,29]$ show good agreement witl our data below $8 \mathrm{MeV}$ but seem to be lower by 15-20\% at higher energies. The shape of the measured excitation function of the ${ }^{57} \mathrm{Fe}(p, n){ }^{57} \mathrm{Co}$ reaction is reproduced well by the calculation; however, small deviation can be seen near the reaction thresliold.

The cross section data for the ${ }^{57} \mathrm{Fe}(p, \alpha){ }^{54} \mathrm{Mn}$ reaction are shown in Fig. 6. Detailed data have been reported only by Levkovskii et al. [30]. Other measurements given in the literature $[24,25,27]$ cover only the energy region above $14 \mathrm{MeV}$. We concentrated our investigation in the vicinity of the threshold. The activity of ${ }^{54} \mathrm{Mn}$ was much lower than that of the other reaction products, mainly due to the long half-life of ${ }^{54} \mathrm{Mn}$ and the low reaction cross section. The experimental data show large deviations, and the errors are large. Our data obtained from several irradiations are consistent. The results given in Ref. [27] are somewhat higher than our values and may be due to the efficiency problem (see above). The data reported by Levkovskii et al. [30], on other hand, are consistently higher than our measurements. The calculated excitation function for the ${ }^{57} \mathrm{Fe}(p, \alpha){ }^{54} \mathrm{Mn}$ reaction is reproduced in Fig. 6. It is close to our measurements, but it should be emphasized that the calculated results for such weak reaction cliannels are sensitive to the level density parameter used in the calculation of cross sections of the strongest reaction channels.

The cross section data for the ${ }^{58} \mathrm{Fe}(p, n){ }^{58} \mathrm{Co}^{m+g}$ process measured in this work and those reported in the literature [24,25] are shown in Fig. 7. Since ${ }^{58}$ Co can be formed only from ${ }^{58} \mathrm{Fe}$, all the data given in Fig. 7 are based on normalization to $100 \%{ }^{58} \mathrm{Fe}$. Obviously both the data sets are in good agreement; the emphasis in the present work was on mesaurement at low energies. Very recently Tims et al. [31] measured cross sections for this

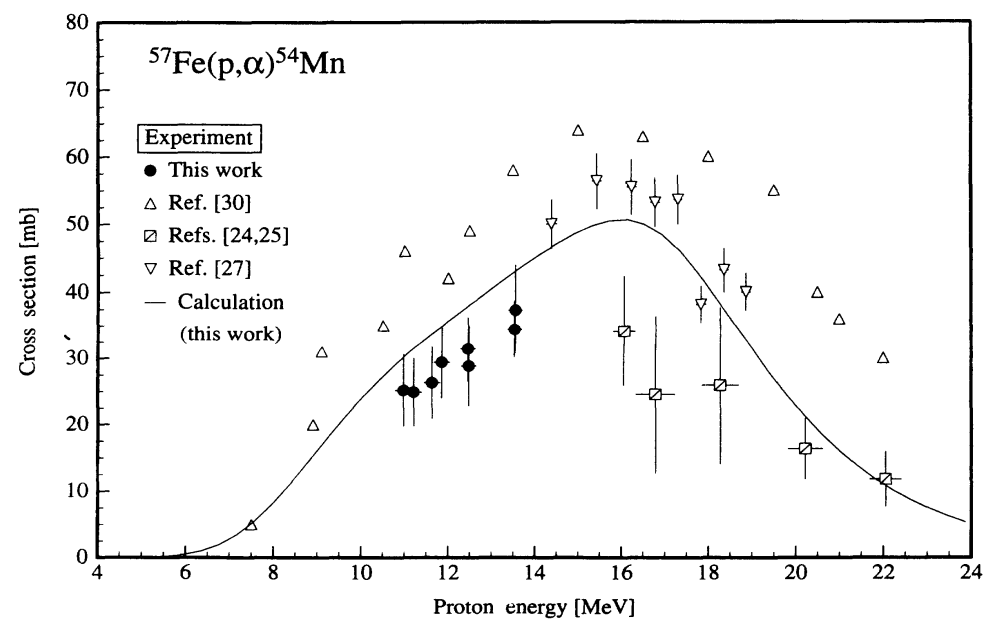

FIG. 6. Measured and calculated cross sections of the ${ }^{57} \mathrm{Fe}(p, \alpha){ }^{54} \mathrm{Mn}$ reaction. 


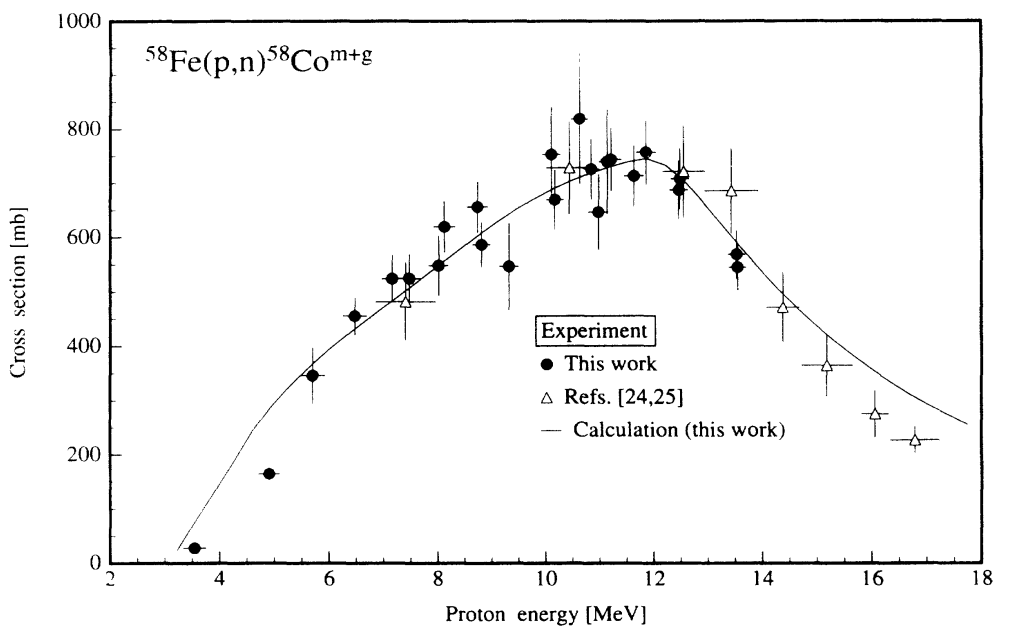

FIG. 7. Measured and calculated cross sections of the ${ }^{58} \mathrm{Fe}(p, n){ }^{58} \mathrm{Co}^{m+g}$ reaction. The experimental data were deduced from measurements on ${ }^{\text {nat }} \mathrm{Fe}$ via normalization to $100 \%{ }^{58} \mathrm{Fe}$.

process in the low energy region of 3.1 to $4.9 \mathrm{MeV}$ using both activation and neutron detection techniques. A 93.23\% enriched ${ }^{58} \mathrm{Fe}$ target was used. Due to the uncertainty in deducing the activation data from the graphical presentation [31], we do not reproduce those data in Fig. 7. In general, however, those results agree with our data. The continous line in Fig. 7 shows the result of our model calculation: the agreement with the experimental data is good. A small deviation at $E_{p} \geq 15 \mathrm{MeV}$ may arise from a too strong preequilibrium contribution.

\section{B. Deuteron induced reactions on iron}

The measured cross section data for the deuteron induced reactions ${ }^{56} \mathrm{Fe}(d, 2 n){ }^{56} \mathrm{Co},{ }^{56} \mathrm{Fe}(d, \alpha){ }^{54} \mathrm{Mn}$, and ${ }^{\text {nat }} \mathrm{Fe}(d, x n){ }^{58} \mathrm{Co}^{m+g}$ are given in Table IV. The data

TABLE IV. Measured cross sections of deuteron induced reactions on iron.

\begin{tabular}{|c|c|c|c|c|c|c|c|}
\hline \multirow{2}{*}{$\begin{array}{c}\text { Deuteron } \\
\text { energy } \\
(\mathrm{MeV})\end{array}$} & \multirow{2}{*}{$\begin{array}{c}\text { Error in } \\
\text { deuteron } \\
\text { energy } \\
(\mathrm{MeV})\end{array}$} & \multicolumn{2}{|c|}{${ }^{\text {nat }} \mathrm{Fe}(d, x n){ }^{58} \mathrm{Co}^{m+g}$} & \multicolumn{2}{|c|}{${ }^{56} \mathrm{Fe}(d, 2 n){ }^{56} \mathrm{Co}$} & \multicolumn{2}{|c|}{${ }^{56} \mathrm{Fe}(d, \alpha){ }^{54} \mathrm{Mn}$} \\
\hline & & $\begin{array}{c}\sigma \\
(\mathrm{mb})\end{array}$ & $\begin{array}{c}\Delta \sigma \\
(\mathrm{mb})\end{array}$ & $\begin{array}{c}\sigma \\
(\mathrm{mb})\end{array}$ & $\begin{array}{c}\Delta \sigma \\
(\mathrm{mb})\end{array}$ & $\begin{array}{c}\sigma \\
(\mathrm{mb})\end{array}$ & $\begin{array}{c}\Delta \sigma \\
(\mathrm{mb})\end{array}$ \\
\hline 3.67 & 0.22 & 1.90 & 0.16 & & & 2.27 & 0.17 \\
\hline 4.45 & 0.22 & 4.02 & 0.30 & & & 6.20 & 0.46 \\
\hline 5.65 & 0.20 & 6.37 & 0.47 & & & 15.3 & 1.1 \\
\hline 7.23 & 0.19 & 6.64 & 0.51 & & & 29.4 & 2.2 \\
\hline 7.38 & 0.22 & 6.51 & 0.38 & & & 28.8 & 1.6 \\
\hline 7.74 & 0.19 & 6.73 & 0.50 & & & 36.5 & 2.7 \\
\hline 7.83 & 0.20 & 6.65 & 0.42 & & & 32.3 & 1.7 \\
\hline 7.87 & 0.21 & & & 0.32 & 0.05 & & \\
\hline 8.00 & 0.24 & & & 0.31 & 0.06 & & \\
\hline 8.17 & 0.20 & & & 0.64 & 0.05 & & \\
\hline 8.26 & 0.20 & 6.08 & 0.37 & 3.01 & 0.21 & 37.5 & 2.2 \\
\hline 8.51 & 0.18 & 6.16 & 0.48 & & & 43.7 & 3.2 \\
\hline 8.62 & 0.18 & & & 10.2 & 0.8 & & \\
\hline 9.05 & 0.18 & 6.16 & 0.49 & 26.6 & 1.9 & 49.8 & 3.7 \\
\hline 9.06 & 0.20 & 5.71 & 0.38 & 20.6 & 1.3 & 44.3 & 2.5 \\
\hline 9.48 & 0.18 & 5.39 & 0.32 & 37.6 & 2.1 & 49.7 & 2.6 \\
\hline 9.85 & 0.18 & 5.55 & 0.37 & 70.6 & 4.5 & 56.3 & 3.3 \\
\hline 10.16 & 0.17 & 5.49 & 0.44 & 69.8 & 4.9 & 57.1 & 4.2 \\
\hline 10.23 & 0.17 & 5.71 & 0.49 & 100.3 & 7.1 & 63.7 & 4.7 \\
\hline 10.56 & 0.18 & 5.24 & 0.34 & 119.0 & 7.2 & 62.3 & 3.5 \\
\hline 10.94 & 0.16 & 5.16 & 0.36 & 138.4 & 7.8 & 62.9 & 2.4 \\
\hline 11.28 & 0.16 & 4.89 & 0.35 & 163.3 & 10 & 63.4 & 2.4 \\
\hline 11.33 & 0.16 & 5.12 & 0.47 & 173.4 & 12 & 67.3 & 5.0 \\
\hline 11.57 & 0.16 & 4.76 & 0.30 & 180.4 & 11 & 64.7 & 3.7 \\
\hline 11.62 & 0.16 & 4.94 & 0.43 & 141.5 & 10 & 62.8 & 4.6 \\
\hline 12.29 & 0.15 & 4.60 & 0.34 & 220.2 & 12 & 66.2 & 3.4 \\
\hline 12.60 & 0.15 & 4.87 & 0.35 & 232.0 & 15 & 63.2 & 3.7 \\
\hline 12.70 & 0.15 & 4.90 & 0.37 & 246 & 17 & 68.4 & 5.0 \\
\hline 12.85 & 0.15 & 4.68 & 0.31 & 248.5 & 15 & 63.1 & 3.6 \\
\hline 12.97 & 0.15 & 4.69 & 0.42 & 233 & 16 & 61.8 & 4.5 \\
\hline
\end{tabular}




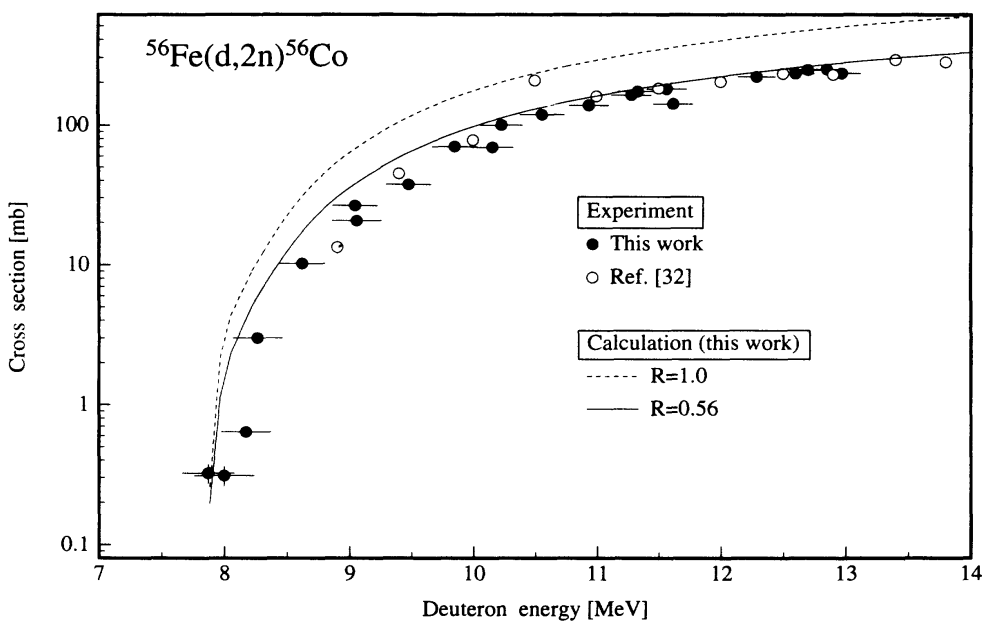

FIG. 8. Measured and calculated cross sections of the ${ }^{56} \mathrm{Fe}(d, 2 n){ }^{56}$ Co reaction. The experimental data were deduced from measurements on ${ }^{\text {nat }} \mathrm{Fe}$ via normalization to $100 \%{ }^{56} \mathrm{Fe}$. for the formation of ${ }^{58} \mathrm{Co}^{m+g}$ refer to ${ }^{\text {nat }} \mathrm{Fe}$ since several reactions could contribute.

The experimental data for the ${ }^{56} \mathrm{Fe}(d, 2 n){ }^{56}$ Co process measured in this work and those reported in the literature [32] are reproduced in Fig. 8 and are in good agreement. Experimental values based on some early measurements $[33,34]$ are not shown since they were presented only in graphical form and have large scaling errors (30-40\%). The results of nuclear model calculations are also shown in Fig. 8. The values are considerably higher than the experimental data. We checked the experimental data carefully. The measurement of radioactivity was done as in the other cases, and the beam current was determined using two different monitor reactions: one internal and the other external. The results on beam current measurements were consistent and also agreed within $\pm 10 \%$ with the values obtained from the Faraday cup. We, therefore, strongly believe that experimental values are reliable and the deviation between experiment and theory is due to some deficiency in the model calculation. Presumably, in the case of deuteron the absorption cross section given by the optical model is an overestinate of the compound absorption cross section. The particle flux goes to a direct channel that is out of the scope of the compound and pre- equilibrium processes and produces the end nuclei through the direct $(d, p n),(d, p)$, and $(d, n)$ processes. A simple approach to take account of this effect is to use a reduction factor in the entrance channel [35]. It can be seen in Fig. 8 that the calculated excitation functions with a reduction factor of $R=0.56$ can give good agreement with the experimental data. Naturally, since this reduction is connected to the entrance channel, all exit channels must show the same reduction, unless some direct contribution exists to a channel.

Figure 9 shows the measured and calculated data for the ${ }^{56} \mathrm{Fe}(d, \alpha){ }^{54} \mathrm{Mn}$ reaction. Our experimental data show good agreement with the literature values [32]. The model calculation was performed using the same reduction factor as that for the ${ }^{56} \mathrm{Fe}(d, 2 n){ }^{56} \mathrm{Co}$ process. A good agreement with the experimental data was obtained.

The experimental data [cf. 32] for the ${ }^{56} \mathrm{Fe}(d, n){ }^{57} \mathrm{Co}$ reaction are given in Fig. 10. Some old values having very large errors, and those presented only as curves [cf. 34] are not shown. We do not report any own measurements on this reaction since we used it as a standard for beam current monitoring and checking the energy loss in the stack. In the nodel calculation we used the same reduc-

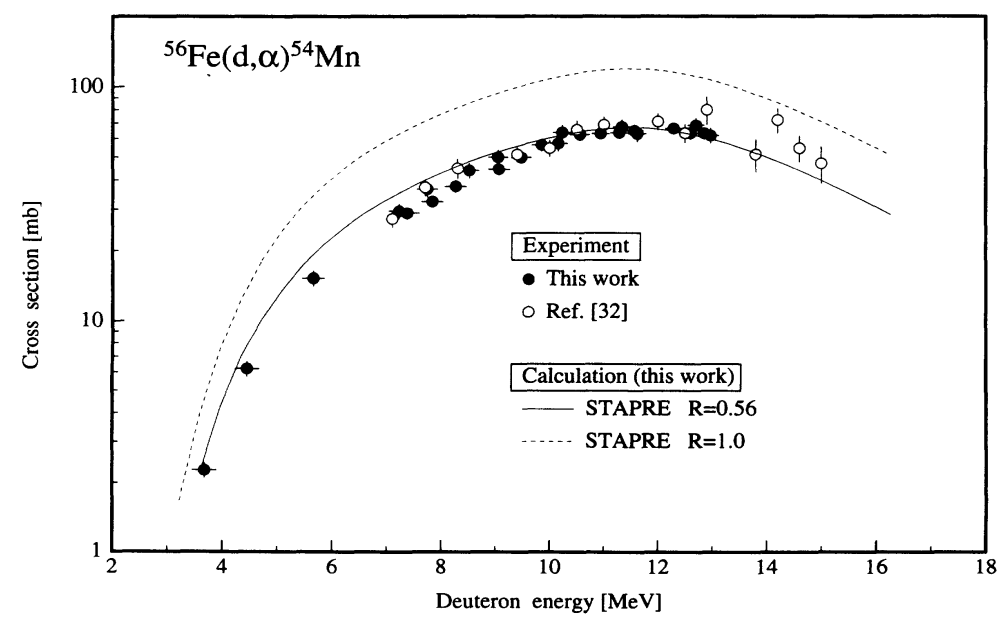

FIG. 9. Measured and calculated cross sections of the ${ }^{56} \mathrm{Fe}(d, \alpha){ }^{54} \mathrm{Mn}$ reaction. Other details were the same as for Fig. 8. 

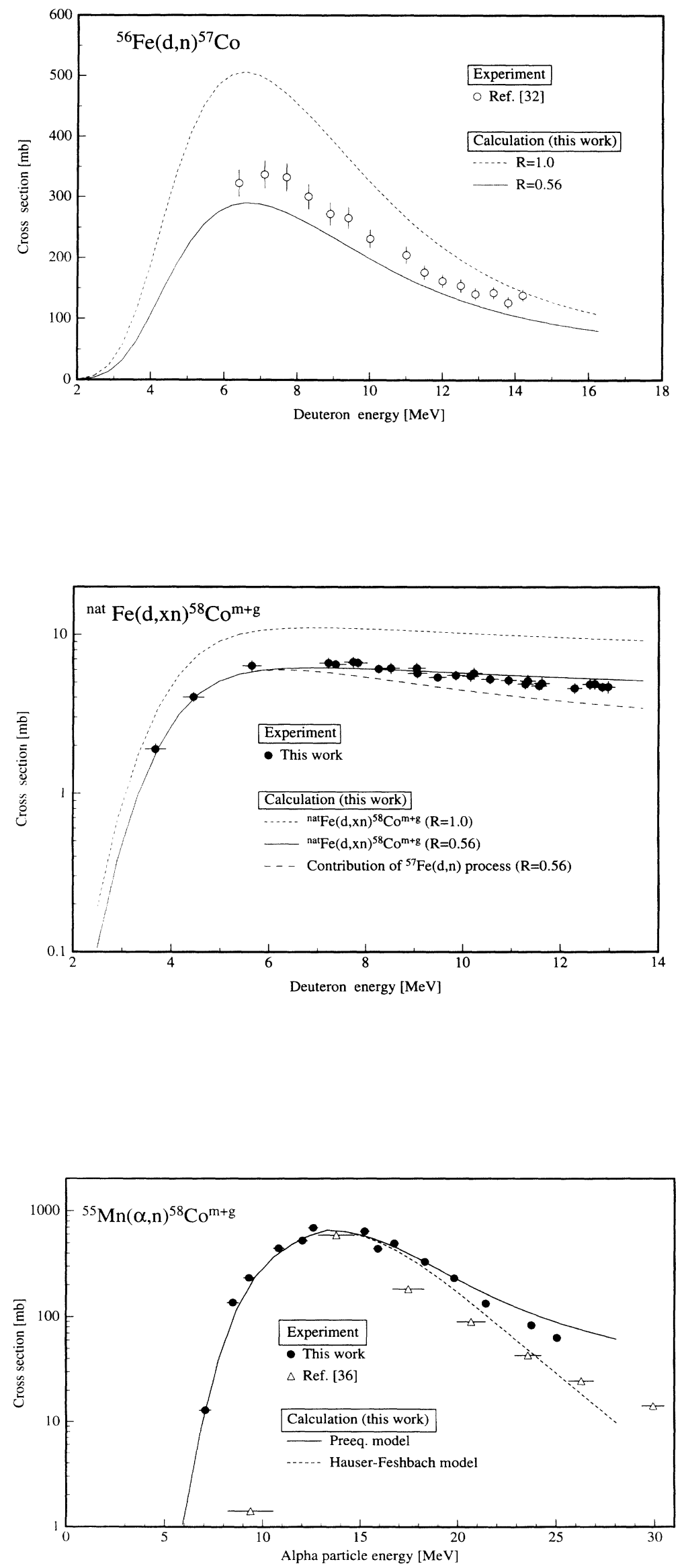

FIG. 10. Measured and calculated cross sections of the ${ }^{56} \mathrm{Fe}(d, n){ }^{57} \mathrm{Co}$ reaction.
FIG. 11. Measured and calculated cross sections of the ${ }^{\text {nat }} \mathrm{Fe}(d, n)^{58} \mathrm{Co}^{m+g}$ process.
FIG. 12. Measured and calculated cross sections of the ${ }^{55} \mathrm{Mn}(\alpha, n){ }^{58} \mathrm{Co}^{m+g}$ process. 


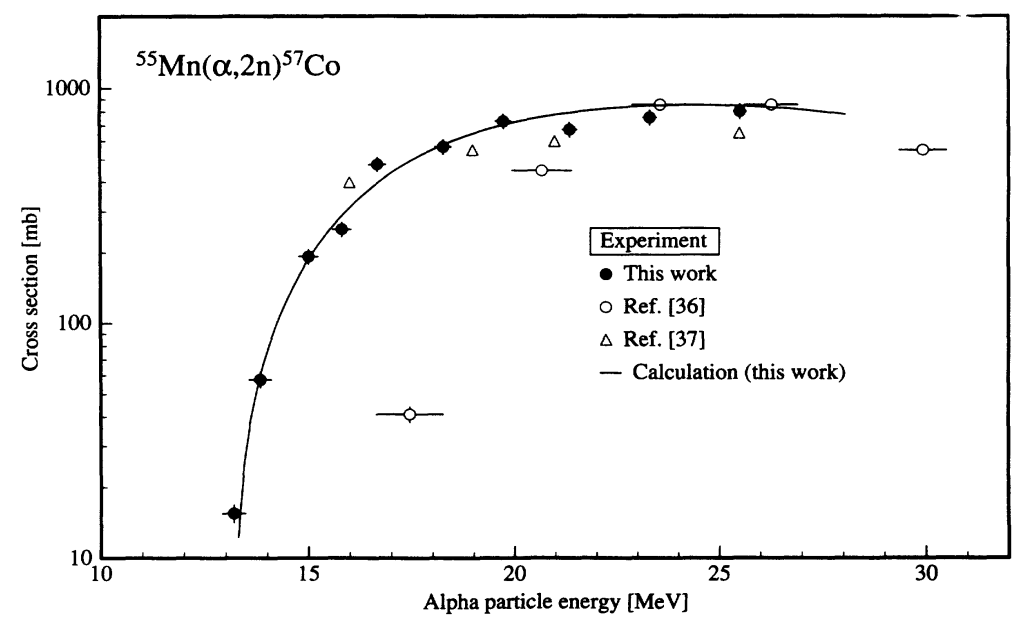

FIG. 13. Measured and calculated cross sections of the ${ }^{55} \mathrm{Mn}(\alpha, 2 n)^{57}$ Co reaction.

tion factor as described above. The results shown in Fig. 10 are $10-15 \%$ lower than the experimental values. This deviation can possibly be attributed to the contribution of the direct $(d, n)$ process. That this contribution is relatively low is presumably due to more favorable $(d, p n)$ breakup or $(d, p)$ stripping.

Figure 11 shows the measured excitation function for the ${ }^{\text {nat }} \mathrm{Fe}(d, x n){ }^{58} \mathrm{Co}^{m+g}$ process. As far as we know, no data exist in the literature. The nuclear model calculation was done using the same reduction factor as for the ${ }^{56} \mathrm{Fe}(d, 2 n){ }^{56} \mathrm{Co}$ process (see above), and the results are given in Fig. 11. Obviously, the measured and cal- culated data are in good agreement; the contribution of the direct process, if any, is then similar to that for the ${ }^{56} \mathrm{Fe}(d, 2 n){ }^{56} \mathrm{Co}$ reaction. The calculational results also show that the major contribution to the formation of ${ }^{58} \mathrm{Co}^{m+g}$ is furnished by the ${ }^{57} \mathrm{Fe}(d, n)$ process.

\section{Alpha-particle induced reactions on manganese}

The measured cross sections for the alpha-particle induced reactions ${ }^{55} \mathrm{Mn}(\alpha, n){ }^{58} \mathrm{Co}^{m+g}$ and ${ }^{55} \mathrm{Mn}(\alpha, 2 n){ }^{57} \mathrm{Co}$ are given in Table V. Figure 12 shows

TABLE V. Measured cross sections of alpha-particle induced reactions on manganese.

\begin{tabular}{|c|c|c|c|c|c|}
\hline \multirow{2}{*}{$\begin{array}{c}\alpha \text {-particle } \\
\text { energy } \\
(\mathrm{MeV})\end{array}$} & \multirow{2}{*}{$\begin{array}{c}\text { Error in } \\
\alpha \text {-particle } \\
\text { energy } \\
(\mathrm{MeV})\end{array}$} & \multicolumn{2}{|c|}{${ }^{55} \mathrm{Mn}(\alpha, n){ }^{58} \mathrm{Co}^{m+g}$} & \multicolumn{2}{|c|}{${ }^{55} \mathrm{Mn}(\alpha, 2 n){ }^{57} \mathrm{Co}$} \\
\hline & & $\begin{array}{c}\sigma \\
(\mathrm{mb})\end{array}$ & $\begin{array}{c}\Delta \sigma \\
(\mathrm{mb})\end{array}$ & $\begin{array}{c}\sigma \\
(\mathrm{mb})\end{array}$ & $\begin{array}{c}\Delta \sigma \\
(\mathrm{mb})\end{array}$ \\
\hline 7.06 & 0.30 & 12.98 & 0.91 & & \\
\hline 8.46 & 0.29 & 136.4 & 9.5 & & \\
\hline 9.29 & 0.28 & 234 & 16 & & \\
\hline 10.80 & 0.27 & 446 & 31 & & \\
\hline 12.02 & 0.25 & 525 & 37 & & \\
\hline 12.58 & 0.24 & 694 & 48 & & \\
\hline 13.20 & 0.28 & & & 15.5 & 1.3 \\
\hline 13.84 & 0.27 & & & 57.6 & 4.2 \\
\hline 15.01 & 0.23 & & & 193 & 14 \\
\hline 15.22 & 0.23 & 642 & 45 & & \\
\hline 15.81 & 0.22 & & & 252 & 18 \\
\hline 15.89 & 0.22 & 441 & 31 & & \\
\hline 16.68 & 0.21 & & & 477 & 33 \\
\hline 16.73 & 0.21 & 493 & 34 & & \\
\hline 18.28 & 0.20 & & & 568 & 40 \\
\hline 18.31 & 0.20 & 333 & 23 & & \\
\hline 19.75 & 0.18 & & & 729 & 51 \\
\hline 19.82 & 0.18 & 232 & 16 & & \\
\hline 21.37 & 0.17 & & & 673 & 47 \\
\hline 21.43 & 0.17 & 133.7 & 9.4 & & \\
\hline 23.33 & 0.16 & & & 756 & 53 \\
\hline 23.76 & 0.16 & 83.8 & 5.9 & & \\
\hline 25.06 & 0.15 & 63.5 & 4.4 & & \\
\hline 25.52 & 0.15 & & & 802 & 56 \\
\hline
\end{tabular}


the measured and calculated excitation function for the ${ }^{55} \mathrm{Mn}(\alpha, n){ }^{58} \mathrm{Co}^{m+g}$ reaction. Our experimental data are significantly higher than those given in Ref. [36], except at the maximum of the excitation function. The data obtained via neutron counting [31], however, agree with our values. The model calculation with preequilibrium emission shows very good agreement with the present measurement up to $20 \mathrm{MeV}$, but above this energy a small deviation occurs which may arise from an overestimation of the preequilibrium fraction. On the other hand the pure Hauser-Feshbach calculation underestimates the cross section in the higher energy range.

Figure 13 depicts the excitation function of the ${ }^{55} \mathrm{Mn}(\alpha, 2 n){ }^{57} \mathrm{Co}$ reaction. Our data are in good agreement with the experimental data given in Ref. [37], while the low energy data given in Ref. [36] deviate considerably both for this reaction and for the $(\alpha, n)$ reaction mentioned above. The model calculation describes well the measured data, especially our values. It should be emphasized that the data near the reaction threshold would not show such a good agreement with the calculation without the application of the mean energy correction described above.

\section{CONCLUSIONS}

From the results presented above it is concluded that the model calculations can describe the measured excitation functions of proton and $\alpha$-particle induced reactions relatively well, provided that appropriate parameters are used in the calculations; global optical model parameters are not good in every case. The deuteron induced reactions can be described only by taking into account in some way the contribution of the direct processes. In cases where several inconsistent experimental data sets are available, the model calculations can possibly help in selecting the best experimental data for evaluation purposes.

\section{ACKNOWLEDGMENTS}

We thank Professor G. Stöcklin and Professor J. Csikai for their active support of this program. This work was done under a German-Hungarian bilateral agreement [No. 13(X237.1)], and it was partly supported by the Hungarian National Science Foundation (OTKA 1734/91).
[1] R. Weinreich, O. Schult, and G. Stöcklin, Int. J. Appl. Radiat. Isot. 25, 535 (1974).

[2] S. M. Qaim, G. Stöcklin, and R. Weinreich, Int. J. Appl. Radiat. Isot. 28, 947 (1977).

[3] H. Piel, S. M. Qaim, and G. Stöcklin, Radiochim. Acta 57, 1 (1992).

[4] Z. Kormány, Nucl. Instrum. Methods Phys. Res. Sect. A 337, 258 (1994).

[5] C. F. Williamson, J. P. Boujot, and J. Picard, "Tables of Range and Stopping Power of Chemical Elements for Charged Particles of Energy 0.5 to $500 \mathrm{MeV}$," Rapport CEA-R 3042, 1966.

[6] R. Gunnink and J. B. Niday, Computerized quantitative analysis by gamma-ray spectrometry. Vol. I, Description of the GAMANAL program, Lawrence Livermore Laboratory, Report UCRL-51061, 1972.

[7] S. Nagy, GAMANAL XT/AT user's manual, Institute of Experimental Physics, Kossuth University, Debrecen, Hungary, Version 1.1, 1989 (unpublished).

[8] M. Uhl and B. Strohmaier, "Computer Code for Particle Induced Activation Cross Section and Related Quantities," Institut für Radiumforschung und Kernphysik Report 76/01, 1976, and Addenda to this report. See also B. Strohmaier and M. Uhl, International Atomic Energy Agency Report IAEA-SMR-43, 1980, p. 313.

[9] O. Bersillon, "SCAT-2: Un programme de modele optique spherique," Centre d'Etudes de Bruyéres-le-Châtel Report, CEA-N-2227, 1981.

[10] F. D. Becchetti and G. W. Greenlees, Phys. Rev. 182, 1190 (1969).

[11] F. G. Perey, Phys. Rev. 131, 745 (1962).

[12] L. McFadden and G. R. Satchler, Nucl. Phys. 84, 177 (1966).

[13] M. Uhl, H. Gruppelaar, H. A. J. van der Kamp, J. Kopecky, and D. Nierop, in Proceedings of the International Conference on Nuclear Data for Science and Tech- nology, Jülich, 1991, edited by S. M. Qaim (SpringerVerlag, Berlin, 1992), p. 924.

[14] A. H. Wapstra and K. Bos, At. Data Nucl. Data Tables 19, 215 (1977).

[15] C. Kalbach, Z. Phys. A 283, 401 (1977).

[16] L. Milazzo-Colli and G. Braga-Marcazzan, Nucl. Phys. A210, 297 (1973).

[17] R. L. Auble, Nuclear Data Sheets 20, 253 (1977); T. W. Burrows and M. R. Bhat, ibid. 47, 1 (1986); P. Anderson, L. P. Ekström, and J. Lyttkens, ibid. 39, 641 (1983); H. Verhuel and R. L. Auble, ibid. 23, 455 (1978).

[18] W. Dilg, W. Schantl, H. Vonach, and M. Uhl, Nucl. Phys. A217, 269 (1973).

[19] I. L. Jenkins and A. G. Wain, J. Inorg. Nucl. Chem. 32, 1419 (1970).

[20] P. Dyer, D. Bodansky, A. G. Seamster, E. B. Norman, and D. R. Maxson, Phys. Rev. C 23, 1865 (1981).

[21] A. S. Iljinov, V. G. Semenov, M. P. Semenova, N. M. Sobolevsky, and L. V. Udovenko, in Interaction of Protons with Targets from $\mathrm{He}$ to $\mathrm{Br}$, edited by $\mathrm{H}$. Schopper, Landolt-Börnstein New Series Vol. 13a (Springer-Verlag, Berlin, 1991).

[22] S. Tanaka and M. Furukawa, J. Phys. Soc. Jpn. 14, 1269 (1959).

[23] J. N. Barrandon, J. L. Debrun, A. Kohn, and R. H. Spear, Nucl. Instrum. Methods 127, 269 (1975).

[24] R. Michel, G. Brinkman, H. Weigel, and W. Herr, Nucl. Phys. A322, 40 (1979).

[25] R. Michel and G. Brinkmann, J. Radioanal. Chem. 59, 467 (1980).

[26] N. C. Schoen, G. Orlov, and R. J. McDonald, Phys. Rev. C 20, 88 (1979).

[27] Zao Wenrong, Lu Hanlin, and Yu Wexiang, Chin. J. Nucl. Phys. 15, 337 (1993).

[28] S. Takács, L. Vasváry, and F. Tárkányi, Nucl. Instrum. Methods Phys. Res. Sect. B 89, 88 (1994). 
[29] C. H. Johnson, A. Galonsky, and J. P. Ultrich, Report No. ORNL-2910, Oak Ridge, 1960.

[30] V. N. Levkovskii, V. F. Reutov, and K. V. Botvin, At. Energ. 69, 180 (1990).

[31] S. G. Tims, A. F. Scott, A. J. Morton, V. Y. Hansper, and D. G. Sargood, Nucl. Phys. A563, 473 (1993).

[32] Tao Zhenlan, Znu Fuying, Qui Huiyuan, and Wang Gonging, At. Energy Sci. Technol. 5, 506 (1983).

[33] W. H. Burgus, G. A. Cowan, J. W. Hadley, W. Hess, T. Shull, M. L. Stevenson, and H. F. York, Phys. Rev. 95, 750 (1954).

[34] J. W. Clark, C. B. Fulmer, and I. R. Williams, Phys. Rev. 179, 1104 (1969).

[35] P. E. Hodgson, Nuclear Reactions and Nuclear Structure (Clarendon Press, Oxford, 1971), p. 293.

[36] B. P. Singh, H. D. Bhardwaj, and R. Prasad, Il Nuovo Cimento 104, 475 (1991).
[37] S. Tanaka, M. Furukawa, T. Mikumo, S. Iwata, M. Yagi, and H. Amano, J. Phys. Soc. Jpn. 15, 545 (1960).

[38] F. Tákányi, F. Szelecsényi, and P. Kopecky, Appl. Radiat. Isot. 42, 513 (1991).

[39] R. Collé, R. Kishore, and J. B. Cumming, Phys. Rev. C 9, 1819 (1974).

[40] P. Kopecky, Int. J. Appl. Radiat. Isot. 36, 657 (1985).

[41] H. F. Roehm, C. J. Verwey, J. Steyn, and W. L. Rautenbach, J. Inorg. Nucl. Chem. 31, 3345 (1969); 32, 2111 (1970).

[42] E. A. Bryant, D. R. F. Cochran, and J. O. Knight, Phys. Rev. 130, 1512 (1963).

[43] N. T. Porile and D. L. Morrison, Phys. Rev. 116, 1193 (1959).

[44] E. Browne and R. B. Firestone, in Table of Radioactive Isotopes, edited by V. S. Shirley (Wiley, New York, 1986). 